\section{Paediatric dentistry}

\section{Implementing 'Was Not Brought'}

Sir, I am a DCT1 working partly in a public dental practice. I first encountered the 'Was Not Brought' (WNB) pathway at a BDA event in 2018. Following Kirby and Harris's article ${ }^{1}$ and the release of the BDA toolkit, ${ }^{2}$ I was keen to implement it. Multiple retrospective audits revealed my practice's compliance with the local pathway for managing children's missed appointments was poor, therefore I sought approval to trial the Was Not Brought Pathway.

When I initially presented the pathway to my colleagues, some concerns were raised. Given the social histories of many of our patients, the three-week wait period to allow the parent to make contact was the point of greatest debate. The families of many of our patients lead chaotic lifestyles, suggesting parents were likely to fail to make contact if the responsibility lay with them. We decided to amend this stage: instead of waiting for the parent to make contact, a new appointment would be posted out for
2-3 weeks' time if phone contact could not be made.

An issue identified during the trial period was that there was no defined way to reappoint the patients after the 'WNB4' stage. Though the clinician is responsible for deciding what action to take, there was no set follow-on other than archiving the record, which was often not appropriate. This posed the risk of losing the most vulnerable patients from the system. A barrier identified by dentists in the practice was that cancelled appointments disappear and leave no record of the child in the day list. Most clinicians work on a part time basis, therefore were often unaware of cancellations made on days when they were not in the practice. If the dentist was not aware an appointment had been cancelled, how could they evaluate the risk to the child? A suggestion to overcome this was to create a logbook of cancelled children's appointments so clinicians could access this information, and repeated cancellations would not go unnoticed.

The overall response to the WNB pathway from my colleagues was positive
- particularly that phone calls to parents immediately after the missed appointment promoted engagement significantly. Use of the pathway also prompted a substantial improvement in information sharing between professionals, as recorded in a second audit cycle.

The WNB pathway is not fully suited to PDS practices in Scotland. However, the improvements seen, and positive feedback received is prompting changes to the existing local pathway. It is my aim that aspects of the WNB pathway will be incorporated in order to successfully promote the welfare and safeguarding of children within our health board.

K. Denholm, Alness, UK

\section{References}

1. Kirby J, Harris J. Development and evaluation of a 'was not brought' pathway: a team approach to managing children's missed dental appointments. Br Dent J 2019; 227: 291-297.

2. British Dental Association. 'Was not brought': New toolkit for safeguarding children who miss appointments. 2019. Available at: https://bda.org/news-centre/ press-releases/Pages/was-not-brought-new-toolkit-forsafeguarding-children-who-miss-appointments.aspx (accessed June 2020). https://doi.org/10.1038/s41415-020-1949-0

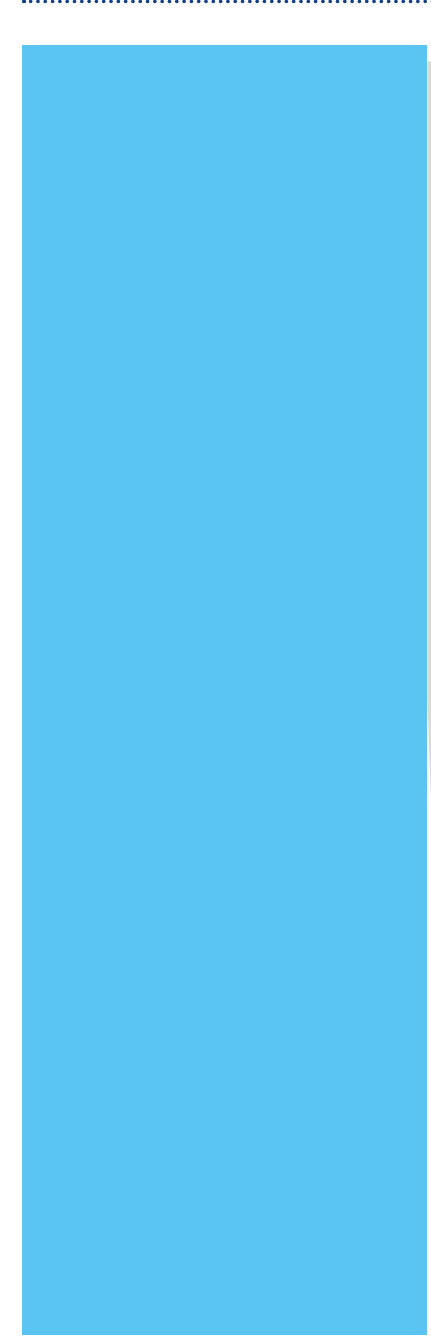

